\title{
Screening for social determinants of health in clinical care: moving from the margins to the mainstream
}

Anne Andermann ${ }^{1,2}$

Correspondence:

anne.andermann@mail.mcgill.ca

${ }^{1}$ St Mary's Research Centre, McGill

University, Montréal, Canada

${ }^{2}$ Department of Family Medicine

and Department of Epidemiology,

Biostatistics and Occupational

Health, McGill University, Montréal,

Canada

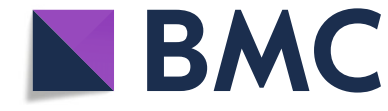

\section{Background}

Determining whether the social determinants influence health is no longer a topic of debate, at least not within the health field. Landmark documents such as the 2008 Closing the Gap report by the World Health Organization Commission on Social Determinants of Health [1] and books such as The Spirit Level by Wilkinson and Pickett [2] have provided substantial evidence to adequately demonstrate that decreasing income, education, social status, and social support is correlated with increased morbidity and premature mortality (also known as the social gradient). Despite the widespread acceptance of the role of social determinants in determining health, whether or not to screen for social determinants of health in clinical care remains a topic of considerable contention.

(c) The Author(s). 2018 Open Access This article is distributed under the terms of the Creative Commons Attribution 4.0 International License (http://creativecommons.org/licenses/by/4.0/), which permits unrestricted use, distribution, and reproduction in any medium, provided you give appropriate credit to the original author(s) and the source, provide a link to the Creative Commons license, and indicate if changes were made. The Creative Commons Public Domain Dedication waiver (http://creativecommons.org/ publicdomain/zero/1.0/) applies to the data made available in this article, unless otherwise stated. 
The two main groups who are less convinced about the value of screening for social determinants of health within clinical care adopt this stance for very different reasons. On the one hand, it has been shown that while many health workers can appreciate the connection between social factors and poor health, common themes explaining their reticence to ask about and address social determinants include being overworked, not knowing how to ask about social determinants or what to do about it once they find out, questioning whether addressing social determinants is part of their role, lacking role models and support in helping patients address the social determinants, being fearful of opening a "Pandora's box" by embarking on this path, and feeling helpless or powerless in the face of such daunting social challenges [3]. A survey conducted by the Robert Wood Johnson Foundation found that four out of five physicians do not feel confident in their capacity to meet their patients' social needs, and they believe this impedes their ability to provide quality care [4].

On the other hand, there are also champions in the field of social determinants who question whether screening is the most appropriate level of intervention. These experts in the field rightly point out that making an impact on social determinants requires broad intersectoral action and whole of government approaches [5]. The factors influencing people's daily living conditions are generally political and structural [6]. These experts therefore question what value, if any, talking to patients about these issues could possibly do to change the larger political and structural forces at play within a society. They consider that action on the social determinants must occur beyond the health sector, but perhaps do not sufficiently appreciate the potential catalyzing role of frontline health workers in advocating and partnering for broader social change, whether at the grassroots community level or at the broader societal level nationally and globally [7]. Indeed, there are many examples of the important influence of physician advocates in many spheres that affect health, from raising awareness on climate change to the 2017 Nobel Peace Prize being awarded to an initiative launched by International Physicians for the Prevention of Nuclear War.

There is growing interest among frontline health workers, particularly, but not limited to, those working in areas such as immigrant and refugee health, caring for homeless and marginally housed persons, inner city health, Indigenous health, social pediatrics, cultural psychiatry, community-oriented primary care and global health, who want to be equipped with evidence-based guidance on how to better care for and support marginalized populations as part of their day-to-day clinical practice. Indeed, with the Lancet Commission on the Education of Health Professionals for the 21st Century highlighting the need for increasing emphasis on social accountability in medical education [8], as well as expanding networks of equity-focused medical educators such as Towards Unity for Health (TUFH), there is a strong core group of health professionals wanting to be more proactive when it comes to addressing social determinants in clinical care.

The purpose of this review is therefore to examine the evidence relating to screening for the social determinants of health in clinical care, including identifying (1) what screening tools currently exist, (2) the potential impact screening can have on improving patient outcomes (i.e., effectiveness), and (3) what factors promote health worker uptake and offer of screening in clinical settings (i.e., adherence).

\section{Methods}

The scoping review followed commonly used methodology as described elsewhere [9]. A search strategy using key search terms relating to social determinants of health and 
screening (Table 1) were used to identify primary and secondary research studies in PubMed (MEDLINE). In total, there were 212 publications identified (Fig. 1). Titles and abstracts were scanned for relevance, and a total of 26 articles were retained. Inclusion criteria consisted of (a) English-language studies from 1970 to the present reporting on the findings of a primary or secondary research study, (b) the main focus of the study relates to screening or systematic case finding of patients with one or more social risk factors (e.g., food insecurity, exposure to violence, poverty), (c) the study involves screening or case finding that is carried out by health workers in a clinical care setting (as opposed to a population-based program), and (d) the publication reports on the types of screening tools used, the impacts of screening, and/or the factors affecting uptake and adherence to screening in clinical care. Exclusion criteria included the following: (a) the main intervention(s) under study do not involve secondary prevention (i.e., screening) or (b) the main aim of screening does not involve identifying social risk. Data extraction was carried out on the 26 retained articles, and findings were grouped according to predetermined and emerging themes using a deductive-inductive approach [10]. The main themes in the deductive framework included the following: (a) how to screen for social risk, (b) what health and social outcomes are impacted as a result of screening, and (c) what are the barriers and facilitators for frontline health workers in adopting social risk screening.

However, as this is an emerging field of research, discussions with key informants and key word searches in Google Scholar were used to identify the grey literature. As well, reference lists of the articles relating to multi-dimensional social risk screening, where there is a dearth of research studies, were scanned to identify other relevant articles using a snowball technique. In this way, a further 41 publications were identified, and data extraction and synthesis was also carried out as detailed above.

Finally, adolopment was used to incorporate evidence that has already been synthesized from previous systematic reviews and meta-analyses. Four evidence-based databases were scanned for high-quality reviews relating to screening for social determinants in clinical care, or related interventions to improve health equity, where the recommendations are to "recommend" or "strongly recommend" the intervention based on moderate- or highquality evidence. The databases searched include The Canadian Task Force on Preventive Health Care, the US Preventive Services Task Force, the NICE UK database, and the Guide to Community Preventive Services. In total, 27 evidence-based reviews were identified, and these interventions are summarized in a separate table.

Table 1 Initial search strategy used for scoping review

(("social determinants of health"[mesh] OR social determinant*[i]) OR ((violence[mesh] OR violence[ti] AND
(health care[ti] OR health services[ti] OR health sector*[ti] OR healthcare[ti])) OR ("Food Supply"[Mesh]) OR (food
insecurit*[ti]) OR (poverty[mesh] OR poverty[ti]) OR (unemployment[mesh] OR unemploy*[ti]) OR (low
income[ti]) OR (underemploy*[ti]) OR ("social isolation"[MeSH Terms]) OR (social exclusion[ti] OR social
isolation[ti] OR socially isolated[ti] OR socially excluded[ti]) OR (support network*[ti] OR social support[ti] OR
social network*[ti]) OR ("Social Environment"[Mesh]) OR ("housing"[MeSH Terms]) OR "homeless persons"[mesh]
OR (homeless*[ti]) OR (hunger[ti]))
AND
("mass screening"[mesh] OR screen*[ti] OR secondary prevention[mesh] OR (prevent*[ti] AND (service*[ti] OR
care[ti] OR healthcare[ti])) OR social history taking[tw] OR preventive practice*[tw])
AND
(practice guidelines as topic[mesh] OR practice guideline[publication type] OR guideline*[ti] OR systematic[sb]
OR evidence informed[ti] OR evidence based[ti] OR recommendation*[ti] OR statement*[ti])




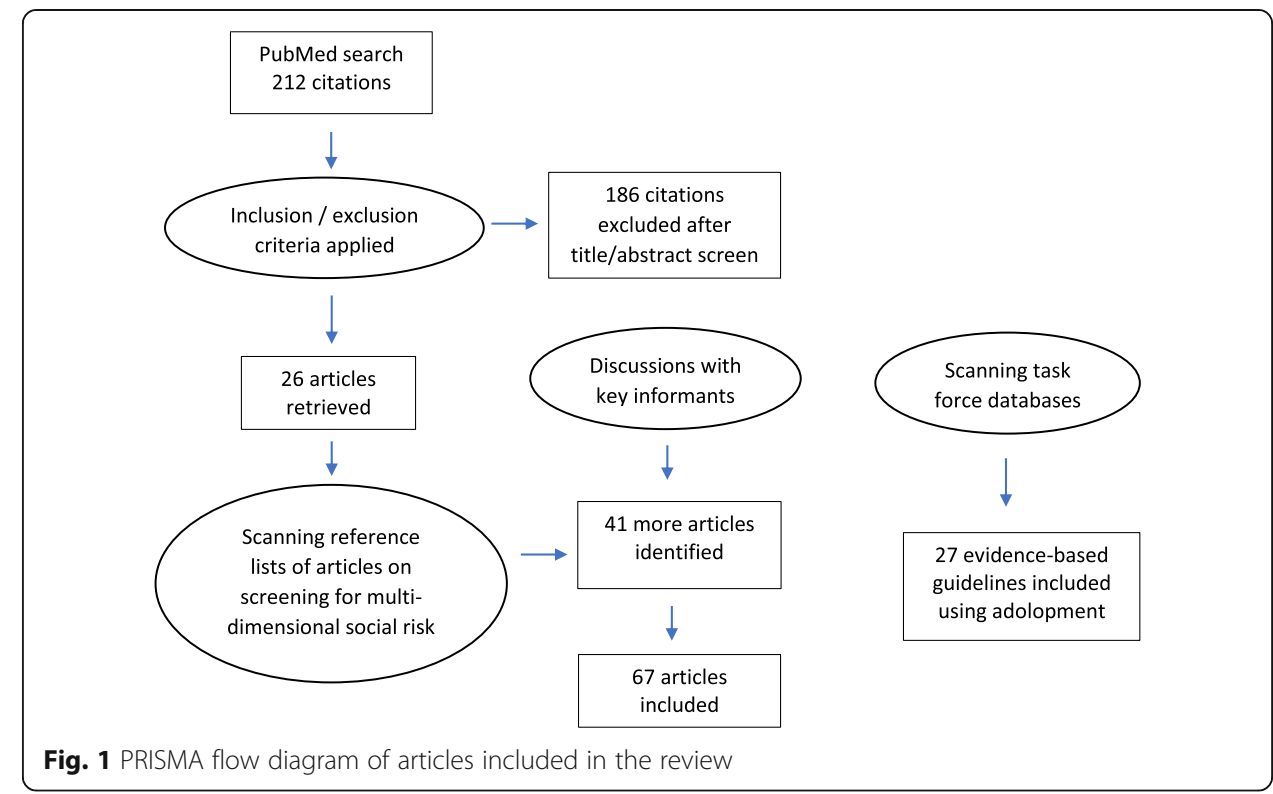

\section{Results}

Much of the growing literature on screening for social determinants of health in clinical practice stems from research in the field of pediatrics, and to a lesser extent in obstetrics and family medicine (Table 2). The majority of these studies were carried out in high-income country contexts, and there is a preponderance of research on screening for violence in particular, with considerably less published in areas such as screening for food insecurity, employment, and housing stability.

The findings of the review are summarized below according to the following main themes: (1) screening tools to identify social determinants in clinical care, (2) evidence of screening effectiveness for improving health and social outcomes, (3) adherence of health professionals to screening guidelines, and (4) whether or not to screen for social determinants of health and under what circumstances (i.e., balancing the benefits versus potential harms).

\section{Screening tools to identify social determinants}

As early as the year 2000, the American College of Obstetricians and Gynaecologists (ACOG) had developed an educational bulletin on perinatal screening and intervention for psychosocial risk factors including barriers to care, frequent moves, safety, food insecurity, substance abuse, partner violence, stress, and unintended pregnancy [11]. The College considered that addressing psychosocial issues is an important part of improving health, that screening should be performed regularly and documented in the patient chart (Table 3), and that an effective system of referrals will increase the likelihood of successful intervention.

Over the last two decades, a growing number of screening tools have been developed to help frontline health workers ask about social determinants of health in clinical care, ranging from identifying food insecurity in specific populations, such as in the elderly [12] or among diabetic patients [13], to screening for violence including adverse 
Table 2 Articles included in the review

\begin{tabular}{|c|c|}
\hline Publication & Topic area \\
\hline Aery et al. 2017 & Screening tools \\
\hline Gallione et al. 2017 & Screening tools \\
\hline Morone et al. 2017 & Screening tools \\
\hline Thomas et al. 2017 & Screening tools \\
\hline Pai et al. 2016 & Screening tools \\
\hline Cohen-Silver et al. 2016 & Screening tools \\
\hline Andermann et al. 2015 & Screening tools \\
\hline Bright et al. 2015 & Screening tools \\
\hline Behforouz et al. 2014 & Screening tools \\
\hline Elbogen et al. 2014 & Screening tools \\
\hline Soc Adol Health and Med, 2013 & Screening tools \\
\hline Vogel, 2013 & Screening tools \\
\hline Hawkins et al. 2012 & Screening tools \\
\hline Bricic et al. 2011 & Screening tools \\
\hline Phelan, 2010 & Screening tools \\
\hline Roffman et al. 2008 & Screening tools \\
\hline Denny, 2007 & Screening tools \\
\hline Olive, 2007 & Screening tools \\
\hline Harley, 2006 & Screening tools \\
\hline Wilson et al. 2006 & Screening tools \\
\hline Savell, 2005 & Screening tools \\
\hline Lapp, 2000 & Screening tools \\
\hline Cohen et al. 1991 & Screening tools \\
\hline Sprague et al. 2016 & Effectiveness_single domain \\
\hline Strong et al. 2016 & Effectiveness_single domain \\
\hline Williams et al. 2016 & Effectiveness_-single domain \\
\hline O'Doherty et al. 2015 & Effectiveness_-single domain \\
\hline O'Doherty et al. 2014 & Effectiveness—single domain \\
\hline Taft et al. 2013 & Effectiveness_-single domain \\
\hline Decker et al. 2012 & Effectiveness_-single domain \\
\hline Taft et al. 2012 & Effectiveness_-single domain \\
\hline Zibowski et al. 2012 & Effectiveness—single domain \\
\hline Feder et al. 2009 & Effectiveness-single domain \\
\hline Killick et al. 2009 & Effectiveness_-single domain \\
\hline Beautrais et al. 2007 & Effectiveness_-single domain \\
\hline Holland and Bultz, 2007 & Effectiveness—single domain \\
\hline Trabold, 2007 & Effectiveness_-single domain \\
\hline Bilukha et al. 2005 & Effectiveness_-single domain \\
\hline Mulvihill, 2005 & Effectiveness_-single domain \\
\hline Taket, 2004 & Effectiveness—single domain \\
\hline Malecha, 2003 & Effectiveness—single domain \\
\hline Wathen et al. 2003 & Effectiveness_-single domain \\
\hline Anderson et al. 2002 & Effectiveness_-single domain \\
\hline Godfrey, 2001 & Effectiveness_-single domain \\
\hline
\end{tabular}


Table 2 Articles included in the review (Continued)

\begin{tabular}{|c|c|}
\hline Publication & Topic area \\
\hline MacMillan, 2000 & Effectiveness_-single domain \\
\hline Sullivan and Bybee, 1999 & Effectiveness_-single domain \\
\hline Chung et al. 2016 & Effectiveness_-multiple domains \\
\hline Naz et al. 2016 & Effectiveness_-multiple domains \\
\hline Garg et al. 2015 & Effectiveness-multiple domains \\
\hline Bigrigg et al. 2005 & Effectiveness_-multiple domains \\
\hline Anderson et al. 2002 & Effectiveness_-multiple domains \\
\hline Grant et al. 2000 & Effectiveness-multiple domains \\
\hline Konijnendijk et al. 2015 & Adherence \\
\hline Miller et al. 2015 & Adherence \\
\hline Konijnendijk et al. 2014 & Adherence \\
\hline Bellini and Quack-Lötscher, 2013 & Adherence \\
\hline Knox and Aspy, 2011 & Adherence \\
\hline O'Campo et al. 2011 & Adherence \\
\hline Reijneveld et al. 2008 & Adherence \\
\hline Mercer et al. 2007 & Adherence \\
\hline Stayton and Duncan, 2005 & Adherence \\
\hline Presley and Robinson, 2002 & Adherence \\
\hline Hakim and Bye, 2001 & Adherence \\
\hline Rapp-Paglicci and Dulmus, 2001 & Adherence \\
\hline Garg et al. 2016 & Whether to screen \\
\hline Gottlieb et al. 2016 & Whether to screen \\
\hline Bayer and Johns, 2016 & Whether to screen \\
\hline Silverstein et al. 2002 & Whether to screen \\
\hline
\end{tabular}

childhood experiences [14], intimate partner violence [15, 16], and elder abuse [17]. For instance, Gallione and colleagues conducted a systematic review of screening tools for elder abuse and identified 11 such tools, some of which had been tested and validated [18]. The authors conclude that the choice of which screening tool to choose depends upon the appropriateness to the clinical setting and context.

In terms of screening for violence, which is generally more developed than other social determinants of health content domains, there are publications on screening as it relates to a variety of settings, from the emergency department [19] to the operating room [20]. As well, there are screening tools not only to identify victims of violence,

Table 3 Sample psychosocial screening tool for prenatal care

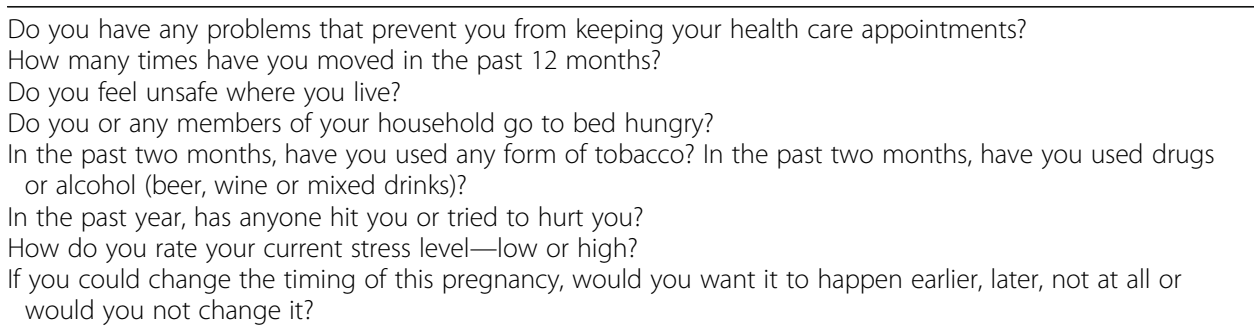


but also potential perpetrators [21, 22]. However, in terms of screening for social determinants of health more broadly, there are often fewer tools that cover multiple domains in a more comprehensive way. For instance, the Poverty Tool, as the name suggests, focuses primarily on screening for financial insecurity using the simple phrase "do you have trouble making ends meet at the end of the month" [23]. This tool is currently in the process of being evaluated in primary care [24].

In the field of pediatrics, Morone from the University of Pennsylvania conducted a recent review of screening tools to identify the social determinants of health in pediatric care; however, relatively few were identified, and among these, the majority focused on a limited number of domains and used heterogeneous approaches for identifying patients at increased risk [25]. Similarly, Pai and colleagues from Ontario, Canada, also conducted a review to identify social risk screening tools for pediatric inpatients, and among 44 instruments identified, $61 \%$ focused on a single social risk theme, only $18 \%$ covered more than 5 themes, and none met the criteria for a valid content and methodologically strong social risk screening instrument for hospitalized children [26]. The authors conclude that more research is needed in this area.

While not a screening tool per se, Behforouz and colleagues propose that what is needed is to train health workers to take a more in depth social history on all patients that includes topics relating to individual characteristics, life circumstances, emotional health, perceptions of health care, health-related behaviors, and access to and utilization of health care (Table 4) [27]. In a similar vein, the CLEAR toolkit which is available for download free of charge in over a dozen languages provides a broad overview of key domains relating to social determinants of health that can be screened for in clinical care (i.e., employment, child care, food insecurity, housing, domestic violence, child maltreatment, discrimination, and isolation) and facilitates mapping out the related referral resources, while encouraging local adaptation of how to ask the questions as well as identifying the most appropriate interventions, based on local knowledge of the specific context [28].

For specific population subgroups, such as adolescents, the HEADSS psychosocial screening tool has been used for several decades and examines (1) the home environment, (2) education and employment, (3) activities, (4) drugs, (5) sexuality, and (6) suicide and depression [29]. Similarly, the Family First screening tool has been used in school-based medical clinics to assess (1) maternal age; (2) education, income, and employment; (3) mental health problems and addictions; (4) parental attachment; (5) marital discord; and (6) social isolation [30]. Thus, a wide range of screening tools are already in existence, though mostly in terms of assessing a single domain of social risk, with some promising examples of multi-domain tools or approaches to social history taking.

\section{Evidence of screening effectiveness on improving patient outcomes}

The evidence of effectiveness of screening for social risk can be divided into two categories: (1) screening for single domains of social risk versus (2) simultaneously screening for multiple domains of social risk. In terms of the former, there is a much larger literature available, as well as several clinical practice guidelines, relating to screening for specific single domains of social risk (e.g., screening for intimate partner violence). However, it is known that social risks tend to cluster. Therefore, intuitively, it makes 
Table 4 Proposed topics for taking a more complete social history

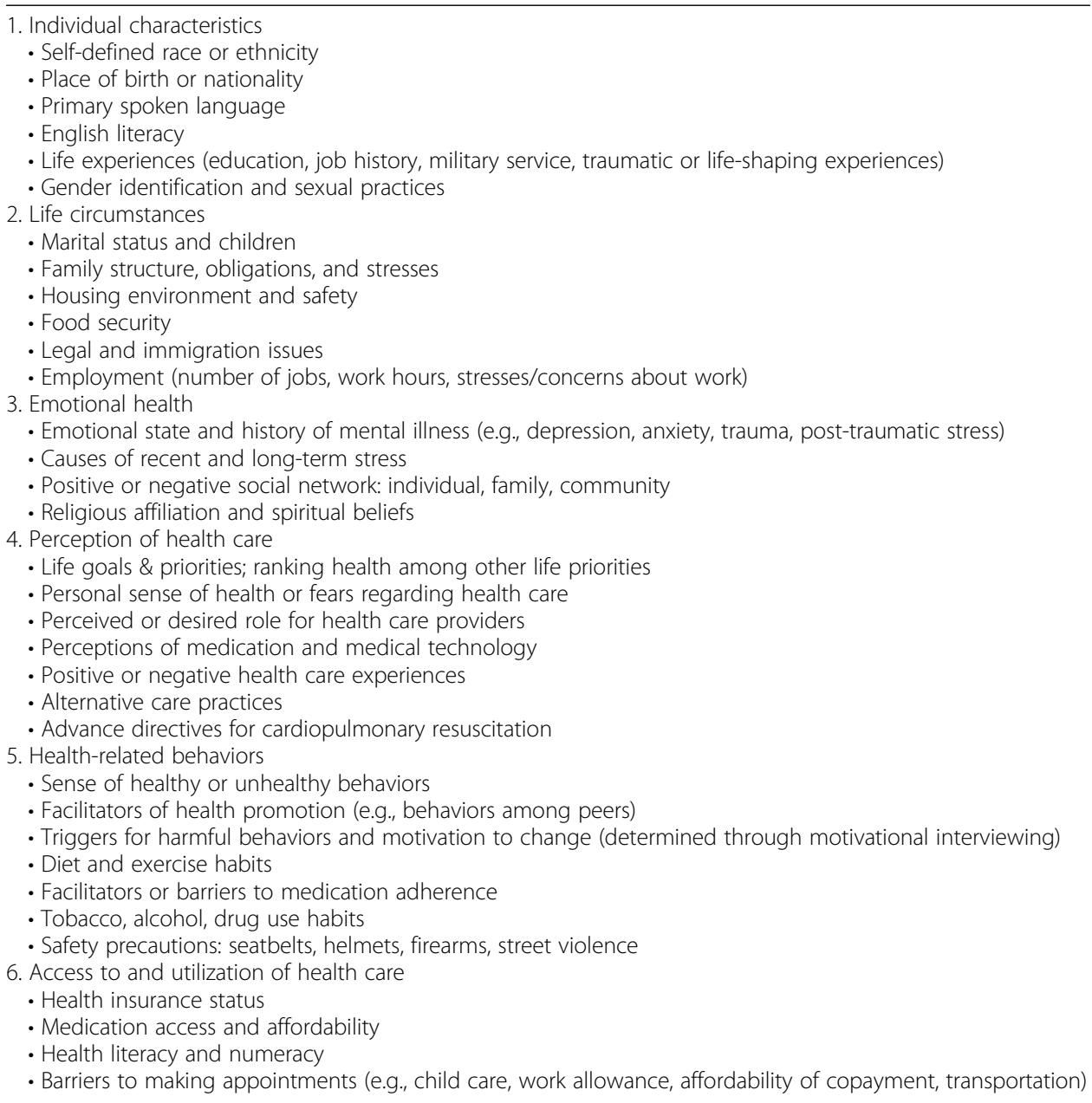
Adapted from reference [27]

sense to simultaneously screen for multiple domains of social risk. This is an approach that has long been used by physicians in the field of social medicine, but a relatively recent focus of research inquiry with regards to mainstream care.

\section{The impact of screening for a single domain of social risk}

There are many evaluations of screening for single domains of social risk and, in particular, a large literature on screening for different types of violence, particularly intimate partner violence [31], as well as suicide (self harm), child abuse, and elder abuse .

While a Cochrane Review did not find sufficient evidence to support an association between screening and reduced harm to women experiencing violence [32], this does not mean that screening is not effective, simply that there is not sufficient evidence at this time to demonstrate an effect [33]. Similarly, an analysis from the UK found that the HITS (Hurts, Insults, Threatens and Screams) scale is a sensitive screening tool able to identify victims of violence in health care settings, most women consider screening for domestic violence to be acceptable, and there is growing evidence of effectiveness for advocacy and psychosocial counselling, nonetheless, universal screening of all women presenting to clinical care in the absence of violence-related concerns or health 
conditions does not yet meet the criteria of the National Screening Committee of the NHS [34].

Not surprisingly, the rates of screening for violence in practice across various health care settings (i.e. prenatal care and pediatrics) are variable [35]. Many in the field advocate for developing more evidence-based approaches to assist women when they do disclose abuse and for greater emphasis on training health professionals to respond appropriately to such disclosures [36]. This is important recognizing that addressing such issues in clinical care can be complex and often raises certain ethical challenges [37] and requires a broader systems approach to ensure patient-centered care, access to appropriate referral pathways, and timely follow-up [38]. Implementation science research is also needed to improve screening uptake and ensure the translation of research findings into routine practice [39].

In terms of current national guidelines, the US Preventive Services Task Force (USPSTF) recommends that health workers screen all women of childbearing age for intimate partner violence and provide or refer women who screen positive to intervention services (Grade B) [40]. The Canadian Task Force on Preventive Health Care (CTFPHC) considers that while there is insufficient evidence to recommend for or against routine universal screening for violence against either pregnant or nonpregnant women (grade I), "clinicians should be alert to signs and symptoms of potential abuse and may wish to ask about exposure to abuse during diagnostic evaluation of these patients" [41]. There is fair evidence to refer women who have spent at least 1 night in a shelter to a structured program of advocacy services [42]. The Community Preventive Services Task Force (CPSTF) as well as the USPSTF and CTFPHC all recommend the use of early childhood home visitation programs based on strong evidence of their effectiveness in reducing child abuse and neglect among high-risk families [43, 44].

In terms of youth violence, a recent systematic review found evidence that health care-based violence intervention programs reduced recidivism as well as health care and criminal justice system costs [45]. In addition, intermediate outcomes included increased service use, positive attitude change, and decreases in violence-related behavior. Regarding suicide, promising interventions likely to be effective in reducing suicidal behaviors are medical practitioner and gatekeeper education, and restriction of access to lethal means of suicide [46].

\section{The impact of simultaneous screening for multiple domains of social risk}

There is a small but growing body of evidence on the impact of screening for social determinants of health more broadly in clinical care. Early research has shown that health workers who feel at ease asking about social determinants of health in clinical care are more likely to report having helped their patients in addressing these issues [47].

The Task Force on Community Preventive Services has conducted systematic reviews of early childhood development interventions and family housing interventions and concluded that these interventions do effectively address sociocultural factors that influence health [48]. The Task Force strongly recommends publicly funded, centerbased, comprehensive early childhood development programs for low-income children aged 3-5 years which are effective in preventing developmental delay. The Task Force also recommends housing subsidy programs for low-income families involving rental 
vouchers for use in the private housing market. This has been shown to improve neighborhood safety and reduce family exposure to violence.

Since this is an emerging area of research, there are mostly primary studies available including non-RCT design [49], as well as a smaller number of RCTs but focusing on diverse types of screening, with a range of different interventions and populations involved, from using patient navigators in primary care [50], to screening and referral for social risk at routine well child visits [51]. There are also a number of clinical practice guides to promote awareness among frontline clinicians, and either encourage screening [52] or at least incorporate a social determinants approach in clinical care and be aware of opportunities when it would be appropriate and good clinical practice to do so.

\section{Adherence of health professionals to screening guidelines}

Even once there are clear national screening guidelines, implementation remains a challenge in this area which can reduce effectiveness in practice. In the Netherlands, while most clinicians were aware of guidelines to identify and address child abuse, fewer than half routinely used the guideline in clinical care, largely because they were not in the habit of doing so [53]. Factors that prevented clinicians from using the guidelines included being unaware of the content, lacking self-efficacy or confidence in being able to apply the guidelines properly, and not having pre-established linkages with referral resources [54]. Four program components that help to increase clinician self-efficacy for screening include institutional support, clear screening protocols, initial and ongoing trainings, and facilitation of access to onsite and/or offsite referral and support services [55]. Adaptation of screening protocols to different clinical settings is also important to assist clinicians in seeing the relevance of screening [56].

Another approach to promote adherence to clinical screening guidelines is to provide incentives and to make referral services more widely available. The US Affordable Care Act includes screening and brief counseling for intimate partner violence as part of required free preventive services for women [57]. There is also evidence that more time to address complex issues, even simply adding a few minutes onto the consultation, is helpful [58], and in primary health care, there is continuity of care and ongoing opportunities to address these issues over time as well as sharing the responsibility for care with a broader clinical team as well as partners working in the community in local NGOs and referral support centers.

\section{Whether or not to screen for social determinants of health}

Already a decade ago, Silverstein and colleagues from Boston University had conducted a review on screening for social determinants of health in pediatric primary care, including identifying factors such as maternal depression, domestic and intimate partner violence, school readiness and eligibility for early learning programs, food insecurity, and housing quality and affordability [59]. The authors concluded that each of these factors is closely linked to patient health outcomes and that the growing body of evidence supports social screening and intervention in primary care, while recognizing the need to continue to develop and refine available screening tools and interventions.

More recently, Garg and colleagues warned about avoiding the unintended consequences of screening for social determinants, particularly in the absence of available referral networks to address identified social needs [60]. This sparked a great deal of debate from supporters of screening who argued that even in the absence of available 
programs and services, screening can help to identify patients who need more support in primary care [61] and can lay the groundwork for the future development of interventions that are better adapted to patient needs [62].

\section{Discussion}

This review has demonstrated that over the last few decades, there has been a growing literature on screening for the social determinants of health in clinical practice. There are an increasing number of screening tools for single and multiple dimensions of social risk and also for specific populations ranging from veterans [63] to the LGBT community [64]. There are also more and more primary research studies and reviews being published that examine the efficacy and effectiveness of screening. For instance, Naz and colleagues [47] found that health workers who have sensitive and caring ways to ask about social determinants were able to open the door to addressing these issues in clinical care. A cluster RCT conducted by Garg and colleagues [51] demonstrated that screening for social determinants of health during well child care visits led to greater referral to social support resources, greater odds of being employed and having child care at 12 months of follow-up, and lower odds of being in a shelter. In addition to improving social outcomes, studies have also shown improvements in health outcomes, such as Strong and colleagues [45] who found that screening for social determinants, and particularly for violence exposure, among youth presenting with injuries led to a reduction in recurrent presentations to clinical care for repeat injuries (i.e., recidivism).

Yet, amassing a body of evidence to demonstrate sufficient benefit in a complex area such as this has resulted in some divergence in national screening recommendations even around single-dimension screening such as screening for intimate partner violence. National recommendations around multi-dimension screening for social risk are not yet available since the evidence base to support such recommendations is highly under-developed at present. More research is still needed in this area to be able to demonstrate whether screening for social risk, and especially for multiple domains of social risk, which require complex and individually tailored interventions, often developed through participatory and community-informed approaches to address local contextual factors [65] and which lead to multiple relevant outcome measures, will succeed in meeting the Wilson and Jungner screening criteria [66]. There is also an ongoing debate regarding the potential unintended consequences of screening which very much depends on how this is done and how well-trained and prepared the clinical staff are and whether referral resources have been sufficiently mapped out. Thus, while adherence to screening in the area of well-defined clinical practice guidelines already demonstrates certain challenges, as one moves towards more complex areas such as screening for social determinants of health, a far more robust evidence base will be needed to generate widespread support and health care culture change in this area.

In light of the current state of the evidence on screening for the social determinants of health, it is important for frontline clinicians to be aware that even when there is insufficient evidence to recommend universal screening of the general population of asymptomatic individuals using specific screening tools and predetermined interventions, clinicians nonetheless require training to know how to ask about social determinants and how to map out referral resources and implement other models of care (e.g., patient navigators) when social risk is relevant to the clinical presentation. Reasons to 
do so are multiple including (1) providing whole-person care rather than focusing only on the disease, (2) reducing missed opportunities for diagnosis by having all the important information in terms of living conditions and social context, (3) reducing "revolving door medicine" and recurrent emergency room visits by understanding and addressing the underlying causes of the presenting health issues, (4) providing more cost-effective care by intervening early and preventing hospitalization, (5) increasing adherence to medication and improving health by prescribing medicines that patients can afford and are therefore more likely to take regularly as prescribed, and (6) providing more trauma-informed and structurally competent care.

For instance, unless health workers routinely ask about exposure to violence in the work up of pelvic pain, they may be missing important opportunities for intervention and may instead embark upon a potentially iatrogenic diagnostic odyssey that misses the main factors underlying the clinical presentation [67]. Similarly, unless clinicians ask their patients whether they will be able to afford the medicines being prescribed, this can lead to medication non-compliance and worsening of the health condition [68]. Just because there may be insufficient evidence to recommend universal screening does not mean that social determinants is unimportant or should be left out of clinical care. Rather, having a heightened awareness of social determinants and enquiring about social history is part of good clinical practice that can lead to better adapted diagnosis and management in a wide range of areas.

Indeed, some would even go so far as to say there is an ethical imperative to act on the social determinants of health in clinical care. According to Rapp-Paglicci and Dulmus, medical centers in the US see 1.4 million serious violent crime victims every year; however very few medical centers evaluate patients beyond physical conditions, and even fewer complete toxicology or psychosocial screens to evaluate for substance abuse and psychological conditions as a result of trauma [69]. Victims of violence are often given medical assistance and discharged without recognition of the serious after effects of trauma for both themselves and their families, as well as the high likelihood of re-victimization..

While the evidence base in this area is still emerging, there already exist numerous examples where there is very strong evidence and widespread consensus regarding interventions that do work to take action on the social determinants of health as summarized in Table 5. Health workers can therefore already get started by incorporating a social determinants lens into their clinical practice and ensuring that patients are able to access these proven interventions ranging from early child home visitation programs for reducing child maltreatment to high school completion programs and tenant-based rental assistance programs.

It is also important to note that screening for social risk is a form of secondary prevention. However, there exists a continuum of strategies to improve the health of populations and reduce inequities [70], from diagnosis and treatment, to the three levels of prevention, to health promotion (i.e., healthy public policies and creating supportive environments for health) [71], as well as broader intersectoral and whole of government action on the social determinants of health [72]. Beyond what can be done at the doctor-patient level, health workers can be important advocates and catalysts of broader changes to create more supportive environments for health and to change social norms, systems, and structures, to prevent these 
Table 5 Evidence-based recommendations to screen for and address social determinants

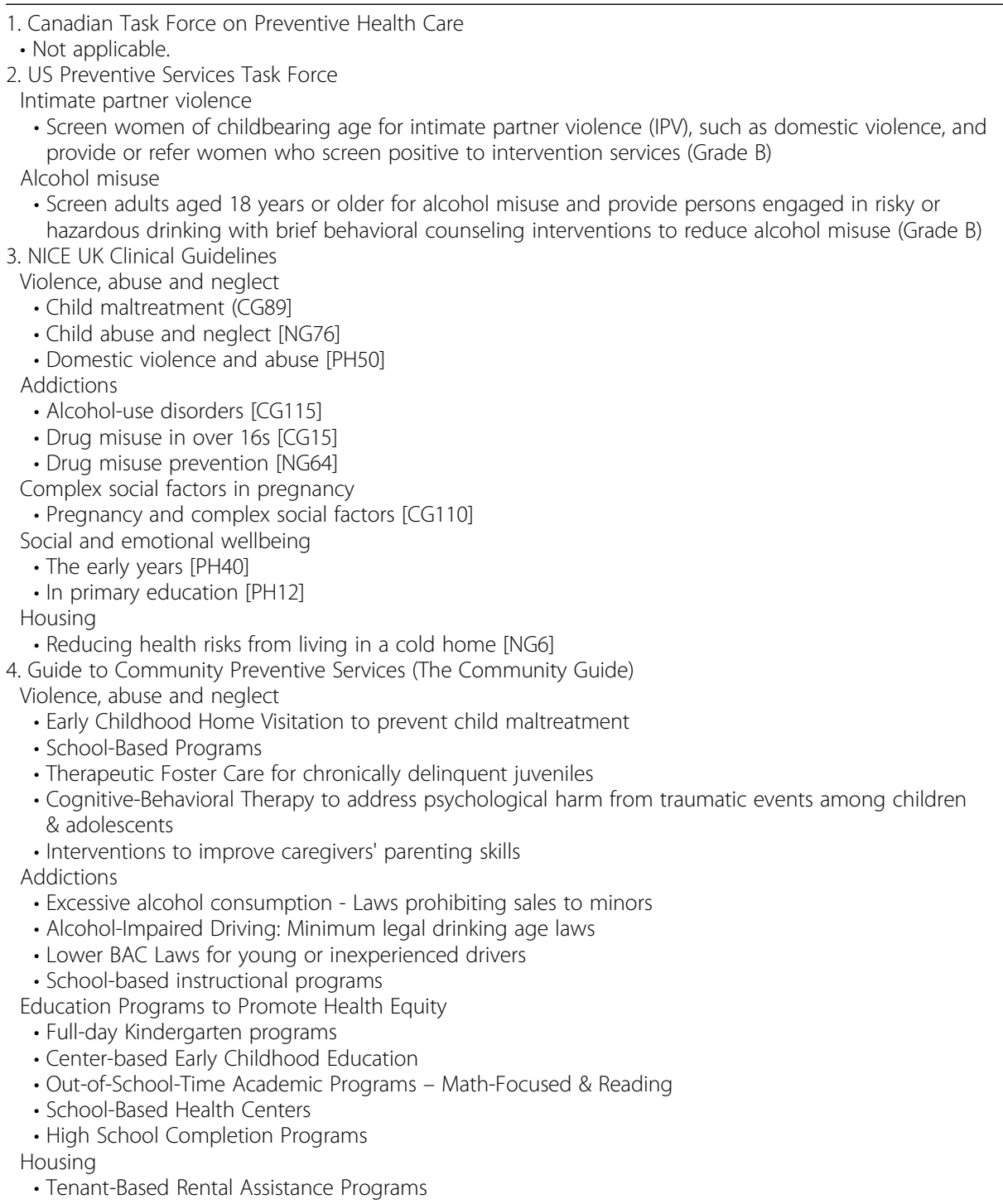

Adapted from the Canadian Task Force on Preventive Health Care [73], the US Preventive Services Task Force [74], the National Institute for Health and Care Excellence UK Clinical Guidelines [75], and The Community Guide [76]

issues from occurring in the first place. This includes informing local and national policy-makers of effective interventions that do exist and ensuring that these are made available for the local population.

\section{Conclusion}

There is a growing body of evidence in support of screening for various aspects of social risk within routine clinical care, as part of a wider continuum of strategies for improving population health and reducing health inequities. At various times in a person's life, everyone may face challenges in one, or often multiple, domains of social risk. Screening for social determinants of health can help to identify patients who may benefit from greater support in one or more areas, thus promoting whole-person care for the entire population, and particularly for those who are marginalized and underserved. 
While there is not always consistency in clinical practice guidelines for singledimension social risk screening (e.g., for identifying intimate partner violence) due to variability of interventions studied and outcomes measured, and while a great deal more research is needed in the area of multi-dimension screening, which is most relevant to the clinical context and meeting patient needs, there already exist many effective and evidence-based interventions to promote health equity, but clinicians would need to identify patients for whom referral to these interventions would be appropriate and may also need to raise awareness and convince local policy-makers to make these interventions available in the local setting. Thus, screening for social determinants of health is an emerging area of clinical practice that still requires a great deal more research and ongoing continuing medical education on how to do this in practice.

Yet, there is increasing traction within the medical field for improving social historytaking and integrating more formal screening for social determinants of health within clinical practice. There is an increasing diversity of screening tools now available, which can be adapted and tailored to the local context, practice population, and needs. There is therefore a great deal that frontline health workers can already do to begin to address social determinants in clinical practice and beyond.

\section{Acknowledgements}

I would like to thank Genevieve Gore, Health Sciences Librarian at McGill University, for her assistance in developing the search strategy for the review.

\section{Funding}

This program of research has been supported by Grand Challenges Canada, Canadian Institutes for Health Research, Fonds de recherche du Québec-Santé, Fédération des médecins spécialistes du Québec and St. Mary's Research Centre, Montréal, Canada.

\section{Author's contributions}

AA drafted and revised the manuscript, approved the final version to be published and agreed to act as guarantor of the work. The author read and approved the final manuscript.

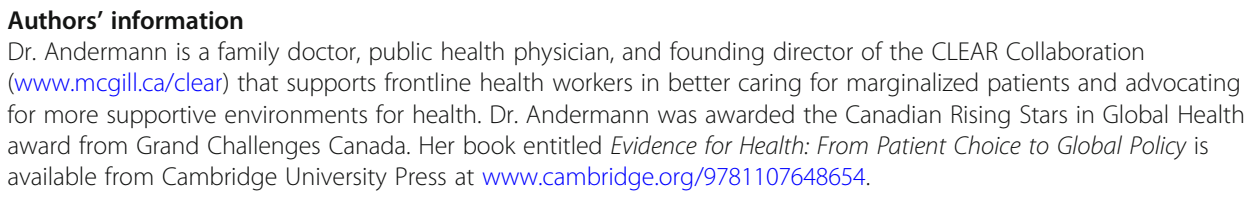

Ethics approval and consent to participate

Not applicable. This manuscript does not report on or involve the use of any animal or human data or tissue.

Competing interests

The author declares that she has no competing interests.

\section{Publisher's Note}

Springer Nature remains neutral with regard to jurisdictional claims in published maps and institutional affiliations.

Received: 13 December 2017 Accepted: 4 April 2018

Published online: 22 June 2018

\section{References}

1. Commission on the Social Determinants of Health. Closing the gap in a generation: health equity through action on the social determinants of health. Geneva: World Health Organization; 2008.

2. Wilkinson RG, Pickett K. The Spirit level: why more equal societies almost always do better. London: Penguin Books; 2010.

3. British Medical Association. Social determinants of health: What can doctors do? London: British Medical Association; 2011. https://www.bma.org.uk/-/media/files/pdfs/working\%20for\%20change/improving\%20health/ socialdeterminantshealth.pdf.

4. Robert Wood Johnson Foundation. Health Care's Blind Side: The Overlooked Connection between Social Needs and Good Health. Princeton, NJ: Robert Wood Johnson Foundation, 2011 https://www.rwjf.org/en/library/research/2011/ 12/health-care-s-blind-side.html. 
5. Dubois A, St-Pierre L, Veras M. A scoping review of definitions and frameworks of intersectoral action. Ciencia Saude Coletiva. 2015;20(10):2933-42.

6. Kindig D, Stoddart G. What is population health? Am J Public Health. 2003;93(3):380-3.

7. Andermann A, CLEAR Collaboration. Taking action on the social determinants of health in clinical practice: a framework for health professionals. CMAJ. 2016;188(17-18):E474-83.

8. Frenk J, Chen L, Bhutta ZA, Cohen J, Crisp N, Evans T, Fineberg H, Garcia P, Ke Y, Kelley P, Kistnasamy B, Meleis A, Naylor D, Pablos-Mendez A, Reddy S, Scrimshaw S, Sepulveda J, Serwadda D, Zurayk H. Health professionals for a new century: transforming education to strengthen health systems in an interdependent world. Lancet. 2010; 376(9756):1923-58.

9. Pham MT, Rajić A, Greig J, Sargeant J, Papadopoulos A, McEwen S. A scoping review of scoping reviews: advancing the approach and enhancing the consistency. Research Synthesis Methods. 2014;5(4):371-385.

10. Crabtree BF, Miller WL. Doing qualitative research: second edition. Thousand Oaks: SAGE Publishing; 1999.

11. Lapp T. ACOG addresses psychosocial screening in pregnant women. The committee on Health Care for Underserved Women of the American College Obstetricians and Gynecologists. Am Fam Physician. 2000;62(12):2701-2.

12. Denny A. Tackling malnutrition among older people in the community. Br J Community Nurs. 2007;12(3):98-102.

13. Thomas B, Fitzpatrick S, Sidani S, Gucciardi E. Developing and implementing a food insecurity screening initiative for patients living with diabetes. Can J Diabetes. 2017; [Epub ahead of print]

14. Bright MA, Thompson L, Esernio-Jenssen D, Alford S, Shenkman E. Primary care pediatricians' perceived prevalence and surveillance of adverse childhood experiences in low-income children. J Health Care Poor Underserved. 2015; 26(3):686-700.

15. Vogel J. Effective gender-based violence screening tools for use in primary health care settings in Afghanistan and Pakistan: a systematic review. East Mediterr Health J. 2013;19(3):219-26.

16. Wilson JS, Lane EM, Gillespie T. Identifying and responding to intimate partner violence in the health care setting. Okla Nurse. 2006;51(2):28-32.

17. Phelan A. Elder abuse and the community nurse: supporting the patient. Br J Community Nurs. 2010;15(10):472-8.

18. Gallione C, Dal Molin A, Cristina FVB, Ferns H, Mattioli M, Suardi B. Screening tools for identification of elder abuse: a systematic review. J Clin Nurs. 2017;26(15-16):2154-76.

19. Olive P. Care for emergency department patients who have experienced domestic violence: a review of the evidence base. J Clin Nurs. 2007;16(9):1736-48.

20. Harley AM. Domestic violence screening: implications for surgical nurses. Plast Surg Nurs. 2006;26(1):24-8.

21. Elbogen EB, Cueva M, Wagner HR, Sreenivasan S, Brancu M, Beckham JC, Van Male L. Screening for violence risk in military veterans: predictive validity of a brief clinical tool. Am J Psychiatry. 2014;171(7):749-57.

22. Roffman RA, Edleson JL, Neighbors C, Mbilinyi L, Walker D. The men's domestic abuse check-up: a protocol for reaching the nonadjudicated and untreated man who batters and who abuses substances. Violence Against Women. 2008;14(5):589-605.

23. Brcic V, Eberdt C, Kaczorowski J. Development of a tool to identify poverty in a family practice setting: a pilot study. Int J Family Med. 2011;2011:812182.

24. Aery A, Rucchetto A, Singer A, Halas G, Bloch G, Goel R, Raza D, Upshur REG, Bellaire J, Katz A, Pinto AD. Implementation and impact of an online tool used in primary care to improve access to financial benefits for patients: a study protocol. BMJ Open. 2017;7(10):e015947.

25. Morone J. An integrative review of social determinants of health assessment and screening tools used in pediatrics. J Pediatr Nurs. 2017:37:22-8

26. Pai N, Kandasamy S, Uleryk E, Maguire JL. Social risk screening for pediatric inpatients. Clin Pediatr. 2016;55(14):1289-94.

27. Behforouz HL, Drain PK, Rhatigan JJ. Rethinking the social history. N Engl J Med. 2014;371(14):1277-9.

28. Andermann A. CLEAR toolkit: helping health workers address the social causes of poor health. Montreal: McGill University; 2015. Available at: https:/www.mcgill.ca/clear/download.

29. Cohen E, Mackenzie R, Yates G. HEADSS, a psychosocial risk assessment instrument: Implications for designing effective intervention programs for runaway youth. Journal of Adolescent Health. 1991;12(7):539-44.

30. Cohen-Silver J, Laher N, Freeman S, Mistry N, Sgro M. Family flRST, an interactive risk screening tool for Families in a School-Based Pediatric Clinic. Clinical Pediatrics. 2016;56(3):217-25.

31. Sprague S, Slobogean GP, Spurr H, McKay P, Scott T, Arseneau E, Memon M, Bhandari M, Swaminathan A. A scoping review of intimate partner violence screening programs for health care professionals. PLoS One. 2016; 11(12):e0168502.

32. O'Doherty L, Hegarty K, Ramsay J, Davidson LL, Feder G, Taft A. Screening women for intimate partner violence in healthcare settings. Cochrane Database Syst Rev. 2015;7:CD007007.

33. Altman DG, Bland JM. Absence of evidence is not evidence of absence. BMJ. 1995;311(7003):485.

34. Feder G, Ramsay J, Dunne D, Rose M, Arsene C, Norman R, Kuntze S, Spencer A, Bacchus L, Hague G, Warburton A, Taket A. How far does screening women for domestic (partner) violence in different health-care settings meet criteria for a screening programme? Systematic reviews of nine UK National Screening Committee criteria. Health Technol Assess. 2009;13(16):iii-v. xi-xiii, 1-113, 137-347

35. Williams JR, Halstead V, Salani D, Koermer N. Intimate partner violence screening and response: policies and procedures across health care facilities. Womens Health Issues. 2016;26(4):377-83.

36. Taket A, Wathen CN, Macmillan H. Should health professionals screen all women for domestic violence? PLoS Med. 2004;1(1):e4.

37. Killick C, Taylor BJ. Professional decision making on elder abuse: systematic narrative review. J Elder Abuse Negl. 2009;21(3):211-38.

38. Miller E, McCaw B, Humphreys BL, Mitchell C. Integrating intimate partner violence assessment and intervention into healthcare in the United States: a systems approach. J Women's Health. 2015;24(1):92-9.

39. Decker MR, Frattaroli S, McCaw B, Coker AL, Miller E, Sharps P, Lane WG, Mandal M, Hirsch K, Strobino DM, Bennett WL, Campbell J, Gielen A. Transforming the healthcare response to intimate partner violence and taking best practices to scale. J Women's Health. 2012;21(12):1222-9. 
40. US Preventive Services Task Force. Intimate Partner Violence and Abuse of Elderly and Vulnerable Adults: Screening. Bethesda: Department of Health and Human Services; 2017. https://www.uspreventiveservicestaskforce. org/.

41. Wathen CN, MacMillan HL, Canadian Task Force on Preventive Health Care. Prevention of violence against women: recommendation statement from the Canadian Task Force on Preventive Health Care. CMAJ. 2003;169(6):582-4.

42. Sullivan CM, Bybee DI. Reducing violence using community-based advocacy for women with abusive partners. J Consult Clin Psychol. 1999;67(1):43-53.

43. Bilukha O, Hahn RA, Crosby A, et al. The effectiveness of early childhood home visitation in preventing violence: a systematic review. Am J Prev Med. 2005;28(2S1):11-39.

44. MacMillan HL, Canadian Task Force on Preventive Health Care. Preventive health care, 2000 update: prevention of child maltreatment. CMAJ. 2000;163(11):1451-8.

45. Strong BL, Shipper AG, Downton KD, Lane WG. The effects of health care-based violence intervention programs on injury recidivism and costs: a systematic review. J Trauma Acute Care Surg. 2016;81(5):961-70.

46. Beautrais A, Fergusson D, Coggan C, Collings C, Doughty C, Ellis P, Hatcher S, Horwood J, Merry S, Mulder R, Poulton R, Surgenor L. Effective strategies for suicide prevention in New Zealand: a review of the evidence. N Z Med J. 2007;120(1251):U2459.

47. Naz A, Rosenberg E, Andersson N, Labonté R, Andermann A, CLEAR Collaboration. Health workers who ask about social determinants of health are more likely to report helping patients: mixed-methods study. Can Fam Physician. 2016:62(11):-684-93.

48. Anderson LM, Shinn C, St CJ, Fullilove MT, Scrimshaw SC, Fielding JE, Normand J, Sanchez-Way R, Richardson T, Centers for Disease Control and Prevention. Community interventions to promote healthy social environments: early childhood development and family housing. A report on recommendations of the Task Force on Community Preventive Services. MMWR Recomm Rep. 2002;51(RR-1):1-8.

49. Bigrigg A, Nandwani R, llett R, Thow C, Lamont M, Bankowska U, Brechin S. Use of a staff administered structured questionnaire to identify relevant life-style issues and social-health determinants in a sexual and reproductive health service. Eur J Contracept Reprod Health Care. 2005;10(1):66-72.

50. Grant C, Goodenough T, Harvey I, Hine C. A randomised controlled trial and economic evaluation of a referrals facilitator between primary care and the voluntary sector. BMJ. 2000;320(7232):419-23.

51. Garg A, Toy S, Tripodis Y, Silverstein M, Freeman E. Addressing social determinants of health at well child care visits: a cluster RCT. Pediatrics. 2015;135(2):e296-304.

52. Chung EK, Siegel BS, Garg A, Conroy K, Gross RS, Long DA, Lewis G, Osman CJ, Jo Messito M, Wade R Jr, Shonna Yin $\mathrm{H}$, Cox J, Fierman AH. Screening for social determinants of health among children and families living in poverty: a guide for clinicians. Curr Probl Pediatr Adolesc Health Care. 2016;46(5):135-53.

53. Konijnendijk AA, Boere-Boonekamp MM, Fleuren MA, Haasnoot ME, Need A. What factors increase Dutch child health care professionals' adherence to a national guideline on preventing child abuse and neglect? Child Abuse Negl. 2015;53:118-27.

54. Konijnendijk AA, Boere-Boonekamp MM, Haasnoot-Smallegange RM, Need A. A qualitative exploration of factors that facilitate and impede adherence to child abuse prevention guidelines in Dutch preventive child health care. J Eval Clin Pract. 2014;20(4):417-24.

55. O'Campo P, Kirst M, Tsamis C, Chambers C, Ahmad F. Implementing successful intimate partner violence screening programs in health care settings: evidence generated from a realist-informed systematic review. Soc Sci Med. 2011;72(6):855-66.

56. Stayton CD, Duncan MM. Mutable influences on intimate partner abuse screening in health care settings: a synthesis of the literature. Trauma Violence Abuse. 2005;6(4):271-85.

57. Ghandour R, Campbell J, Lloyd J. Screening and counseling for intimate partner violence: A vision for the future. J Womens Health. 2015;24(1):57-61.

58. Mercer SW, Fitzpatrick B, Gourlay G, Vojt G, McConnachie A, Watt GC. More time for complex consultations in a high-deprivation practice is associated with increased patient enablement. Br J Gen Pract. 2007;57(545):960-6.

59. Silverstein M, Conroy K, Sandel M. Screening for social determinants of health in pediatric primary care. Pediatr Ann. 2008:37(11):740-6.

60. Garg A, Boynton-Jarrett R, Dworkin PH. Avoiding the unintended consequences of screening for social determinants of health. JAMA. 2016;316(8):813-4.

61. Gottlieb L, Fichtenberg C, Adler N. Screening for social determinants of health. JAMA. 2016;316(23):2552.

62. Bayer R, Johns DM. Screening for social determinants of health. JAMA. 2016;316(23):2551-2.

63. Hawkins EJ, Grossbard J, Benbow J, Nacev V, Kivlahan DR. Evidence-based screening, diagnosis, and treatment of substance use disorders among veterans and military service personnel. Mil Med. 2012;177(8 Suppl):29-38.

64. Society for Adolescent Health and Medicine. Recommendations for promoting the health and well-being of lesbian, gay, bisexual, and transgender adolescents: a position paper of the Society for Adolescent Health and Medicine. J Adolesc Health. 2013;52(4):506-10.

65. Mckernan McKay M, Alicea S, Elwyn L, McClain ZR, Parker G, Small LA, Mellins CA. The development and implementation of theory-driven programs capable of addressing poverty-impacted children's health, mental health, and prevention needs: CHAMP and CHAMP+, evidence-informed, family-based interventions to address HIV risk and care. J Clin Child Adolesc Psychol. 2014;43(3):428-41.

66. Andermann A, Blancquaert I, Beauchamp S, Déry V. Revisiting Wilson and Jungner in the genomic age: a review of screening criteria over the past 40 years. Bull World Health Organ. 2008;86(4):317-9.

67. MacMillan HL, Wathen CN. Violence against women: integrating the evidence into clinical practice. CMAJ. 2003; 169(6):570-1.

68. Bloch G, Rozmovits L, Giambrone B. Barriers to primary care responsiveness to poverty as a risk factor for health. BMC Fam Pract. 2011;12:62.

69. Rapp-Paglicci L, Dulmus CN. Ignoring the violence: healthcare policy recommendations for the prevention of community violence re-victimization. J Health Soc Policy. 2001;14(2):45-53. 
70. Andermann A. Evidence for health: from patient choice to global policy. Cambridge: Cambridge University Press; 2013.

71. World Health Organization. Ottawa Charter for Health Promotion. Geneva: World Health Organization; 1986. http:// www.who.int/healthpromotion/conferences/previous/ottawa/en/.

72. Lawless AP, Williams C, Hurley C, Wildgoose D, Sawford A, Kickbusch I. Health in all policies: evaluating the South Australian approach to intersectoral action for health. Can J Public Health. 2012;103(7 Suppl 1):eS15-9.

73. Canadian Task Force on Preventive Health Care. https://canadiantaskforce.ca/. Accessed 17 Apr 2018.

74. US Preventive Services Task Force. https://www.uspreventiveservicestaskforce.org/. Accessed 17 Apr 2018.

75. National Institutes for Health and Care Excellence. https:/www.nice.org.uk. Accessed 17 Apr 2018.

76. The Community Guide. https://www.thecommunityguide.org/. Accessed 17 Apr 2018.

Ready to submit your research? Choose BMC and benefit from:

- fast, convenient online submission

- thorough peer review by experienced researchers in your field

- rapid publication on acceptance

- support for research data, including large and complex data types

- gold Open Access which fosters wider collaboration and increased citations

- maximum visibility for your research: over $100 \mathrm{M}$ website views per year

At BMC, research is always in progress.

Learn more biomedcentral.com/submissions 